\title{
The scalars from the topcolor scenario and the spin correlations of the top pair production at the LHC
}

\author{
Chong-Xing Yue, Ting-Ting Zhang, Jin-Yan Liu \\ Department of Physics, Liaoning Normal University, Dalian 116029, P. R. China *
}

December 4, 2018

\begin{abstract}
The topcolor scenario predicts the existences of some new scalars. In this paper, we consider the contributions of these new particles to the observables, which are related to the top quark pair $(t \bar{t})$ production at the LHC. It is found that these new particles can generate significant corrections to the $t \bar{t}$ production cross section and the $t \bar{t}$ spin correlations.
\end{abstract}

PACS numbers: 12.60.Cn, 12.15.Lk, 13.38.Dg

*E-mail:cxyue@lnnu.edu.cn 


\section{Introduction}

Searching for the standard model (SM) Higgs boson is one of the main tasks of the large hadron collider (LHC), which has a considerable capability to discover and measure almost all of its quantum properties [1]. However, if the LHC finds evidence for a new scalar state, it may not necessarily be the SM Higgs boson. Most of the new physics models beyond the SM predict the existence of new scalar states. These new particles may produce contributions to some physical observables. Thus, studying the possible signals of the new scalar states at the current and near future high energy collider experiments is of special interest, which will be helpful to test the SM and further to differentiate various kinds of new physics models.

To completely avoid the problems of triviality and hierarchy arising from the elementary Higgs field in the SM, various kinds of dynamical electroweak symmetry breaking (EWSB) models have been proposed. Among these new physics models, topcolor scenario is attractive because it can explain the large top quark mass and provides a new possible EWSB mechanism [2]. The main features of this kind of model are: EWSB is mainly driven by the technicolor interaction, the masses of the light quarks and leptons and a very small portion of the top quark mass are generated by the extended technicolor interaction. The topcolor interaction gives the main part of the top quark mass and makes small contributions to EWSB. Topcolor scenario generally predicts a number of new scalar states at the electrowake scale: three top-pions $\left(\pi_{t}^{ \pm}, \pi_{t}^{0}\right)$, a top-Higgs boson $\left(h_{t}^{0}\right)$, and a techni-Higgs boson $\left(h_{t c}^{0}\right)$. Some of these new particles couple preferentially to the third generation fermions and might produce significant contributions to the physical observables related to the top quark.

The top quark with a mass of the order of the electroweak scale is the heaviest elementary particle discovered to date, which is singled out to play a key role in probing the new physics beyond the SM [3]. An important property of the top quark is that, compared to lighter quarks, its lifetime is extremely short so that its properties are not polluted by the hadronization process. In the absence of hard gluon radiation, top quark polarization 
is conserved, its spin information can be transferred into its decay products. This information can be used to study the Lorentz structure of interaction vertices involved in top quark production and decay.

At the LHC, a large number of top quarks will be produced every year. This fact makes that it is possible to measure the observables that depend on the top quark spin and the top quark properties with high precision at this facility, which will provide a good probe for tests of the SM and for searches of new physics beyond the SM. For hadronic top quark pair $(t \bar{t})$ production, spin correlations have been extensively studied in the quantum choromodynamics (QCD) $[4,5,6]$. If the new particles have sizable couplings to the top quark, then they can produce contributions to the $t \bar{t}$ spin correlations. Effects of new physics on the $t \bar{t}$ spin correlations have been studied at the $e^{+} e^{-}$collider [7], the photon collider [8], and the hadron colliders [9]. In this paper, we consider the contributions of the scalar particles predicted by the topcolor scenario to the $t \bar{t}$ production at the LHC and further discuss their effects on the $t \bar{t}$ spin correlations. We find that these new particles can indeed give significant contributions to the $t \bar{t}$ production cross section and sizable deviation of the $t \bar{t}$ spin correlations from the SM prediction are possible with reasonable values of the free parameters.

The topcolor-assisted technicolor (TC2) model [10] is one of the phenomenologically viable models, which has almost all essential features of the topcolor scenario. So, in the rest of this paper, we will give our numerical results in detail under this model. In the following section, we will give the relevant formula for our calculation. The numerical results and a short discussion are shown in the last section.

\section{The relevant calculation formula}

For the TC2 model [10], technicolor (TC) interactions play a main role in breaking the electroweak symmetry. Topcolor interaction makes small contributions to $E W S B$, and gives rise to the main part of the top quark mass, $(1-\varepsilon) m_{t}$, with the parameter $\varepsilon \ll 1$. 
Thus, there is the relation

$$
\nu_{\pi}^{2}+F_{t}^{2}=\nu_{w}^{2}
$$

where $\nu_{\pi}$ represents the contributions of TC interactions to EWSB, $\nu_{w}=\nu / \sqrt{2} \simeq$

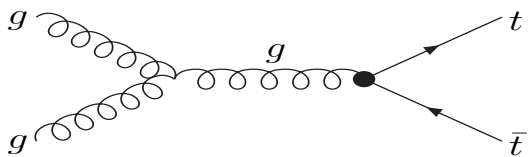

(a)

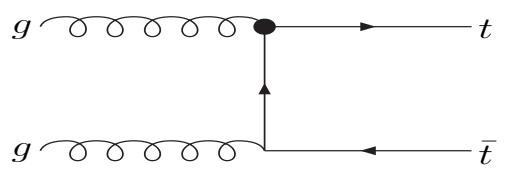

(c)

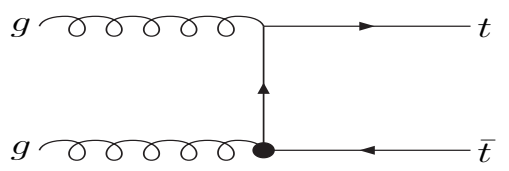

(e)

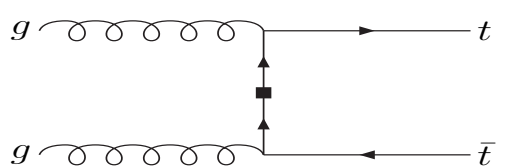

(g)

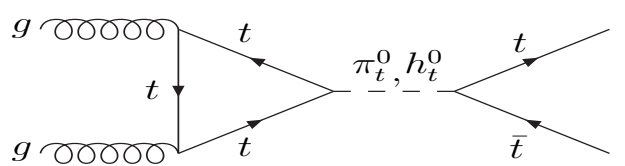

(i)

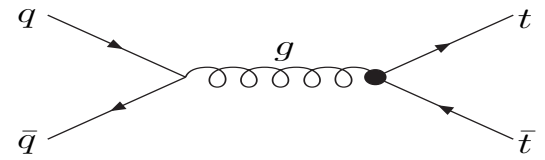

(b)

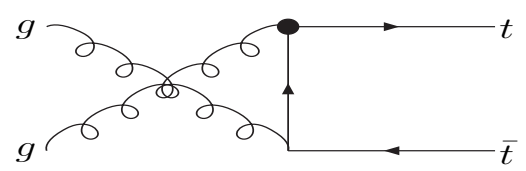

(d)

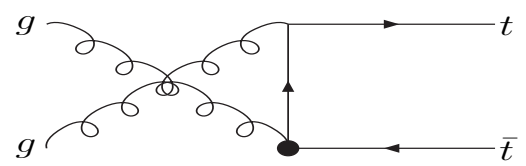

$(f)$

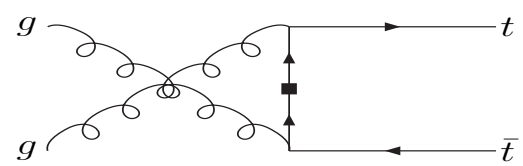

(h)

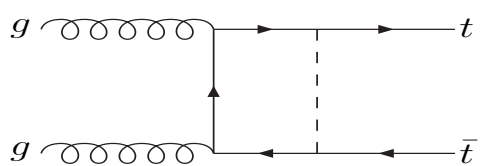

( $j)$

Figure 1: Feynman diagrams for the partonic processes $i \rightarrow t \bar{t}(i=g g$ and $q \bar{q})$ in the TC2 model.

$174 G e V$. Here $F_{t} \simeq 50 G e V$ is the physical top-pion decay constant, which can be esti- 
mated from the Pagels-Stokar formula. The scalar particles predicted by the TC2 model are bound-states of the techni-fermions and of top quark, bottom quark, which are the techni-Higgs boson $\left(h_{t c}^{0}\right)$, top-pions $\left(\pi_{t}^{ \pm}, \pi_{t}^{0}\right)$, and top-Higgs boson $\left(h_{t}^{0}\right)$.

As it is well known that, in the TC2 model, topcolor interaction is not flavor-universal and mainly couple to the third generation fermions. Thus, the couplings of the toppions $\left(\pi_{t}^{ \pm}, \pi_{t}^{0}\right)$ to the three family fermions are non-universal and they have large Yukawa couplings to the third family. The couplings of these new scalar particles to the third family quarks can be written as $[10,11]$ :

$$
\begin{array}{r}
\frac{m_{t}}{\sqrt{2} F_{t}} \frac{\sqrt{\nu_{w}^{2}-F_{t}^{2}}}{\nu_{w}}\left(i K_{U L}^{t t *} K_{U R}^{t t} \bar{t}_{L} t_{R} \pi_{t}^{0}+\sqrt{2} K_{U R}^{t t *} K_{D L}^{b b} \bar{t}_{R} b_{L} \pi_{t}^{+}+\right. \\
\left.i K_{U L}^{t t *} K_{U R}^{t c} \bar{t}_{L} c_{R} \pi_{t}^{0}+\sqrt{2} K_{U R}^{t c *} K_{D L}^{b b} \bar{c}_{R} b_{L} \pi_{t}^{+}+\text {h.c. }\right),
\end{array}
$$

where the factor $\sqrt{\nu_{w}^{2}-F_{t}^{2}} / \nu_{w}$ reflects the effect of the mixing between the top-pions and the would be Goldstone bosons [12]. To yield a realistic form of the $C K M$ matrix $V_{C K M}$, it has been shown that the values of the matrix elements $K_{U L(R)}^{i j}$ can be taken as [13]:

$$
K_{U L}^{t t} \approx K_{D L}^{b b} \approx 1, \quad K_{U R}^{t t} \approx 1-\varepsilon, \quad K_{U R}^{t c} \leq \sqrt{2 \varepsilon-\varepsilon^{2}}
$$

The relevant couplings for the top-Higgs boson $\left(h_{t}^{0}\right)$ are similar to those of the neutral top-pion $\pi_{t}^{0}$ [13]. However, for the techni-Higgs boson $h_{t c}^{0}$, it is not this case. Its coupling to the top quark pair $t \bar{t}$ is very small, which is proportionate to the factor $\varepsilon / \sqrt{2}[14]$. Furthermore, the mass of $h_{t c}^{0}$ is at the order of $1 T e V$. Thus, compared to the top-Higgs and the top-pions, the contributions of the techni-Higgs to the $t \bar{t}$ production can be neglected.

At hadron colliders, the top quark pair $t \bar{t}$ is produced through the partonic processes of quark-antiquark pair annihilation and gluon fusion:

$$
i \rightarrow t+\bar{t}, \quad i=g g, \quad q \bar{q} .
$$

Where $q$ denotes $u, c, d, s$, or $b$ quark. It is well known that the former is the dominate process at the LHC. In the context of the TC2 model, the Feynman diagrams for these partonic processes are depicted in Fig.1. The black dot in Fig.1 represents the effective 
$g t \bar{t}$ vertex induced by the new scalar particles $\left(\pi_{t}^{ \pm}, \pi_{t}^{0}, h_{t}^{0}\right)$, as shown in Fig.2. Note that the boson in each loop denotes a neutral top-pion, top-Higgs or a charged top-pion, while the fermion in each loop can be a top or bottom quark depending on the involved boson being neutral or charged.

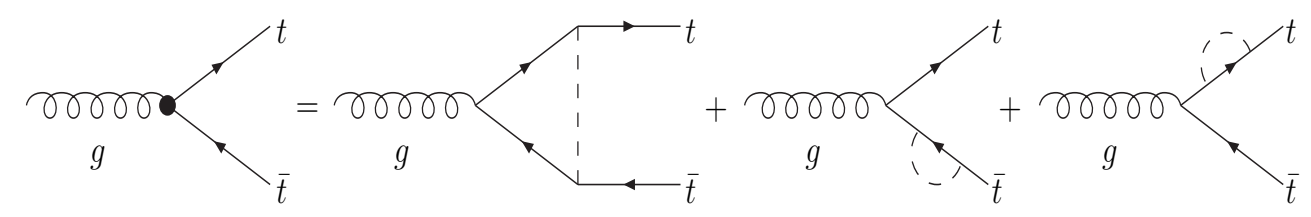

Figure 2: Feynman diagrams for the effective vertex $g t \bar{t}$ in the TC2 model.

The invariant amplitudes for the partonic processes $g\left(p_{1}\right) g\left(p_{2}\right) \rightarrow t\left(k_{1}, s_{t}\right) \bar{t}\left(k_{2}, s_{\bar{t}}\right)$ and $q\left(p_{1}^{\prime}\right) \bar{q}\left(p_{2}^{\prime}\right) \rightarrow t\left(k_{1}, s_{t}\right) \bar{t}\left(k_{2}, s_{\bar{t}}\right)$ contributed by the neutral top-pion or the top-Higgs can be written as follows:

$$
\begin{aligned}
M_{a}= & g_{s}^{2} T^{a} f^{a b c}\left(p_{1}-p_{2}\right)_{\sigma} \epsilon^{\mu}\left(p_{1}\right) \epsilon^{\nu}\left(p_{2}\right) \frac{g_{\mu \nu}}{\left(p_{1}+p_{2}\right)^{2}} \bar{u}\left(k_{1}, s_{t}\right)\left[A_{a} \gamma^{\sigma}+i B_{a} \sigma^{\sigma \alpha}\left(p_{1}+p_{2}\right)_{\alpha}\right. \\
+ & \left.C_{a}\left(\gamma^{\sigma}-\frac{2 m_{t}}{\left(p_{1}+p_{2}\right)^{2}}\left(p_{1}+p_{2}\right)^{\sigma}\right)\right] \nu\left(k_{2}, s_{\bar{t}}\right) ; \\
M_{b}= & -i g_{s}^{2} T^{a} T^{b} \bar{v}\left(p_{1}^{\prime}\right) \gamma^{\mu} u\left(p_{2}^{\prime}\right) \frac{g_{\mu \nu}}{\left(p_{1}^{\prime}+p_{2}^{\prime}\right)^{2}} \bar{u}\left(k_{1}, s_{t}\right)\left[A_{b} \gamma^{\nu}+i B_{b} \sigma^{\nu \alpha}\left(p_{1}^{\prime}+p_{2}^{\prime}\right)_{\alpha}\right. \\
& \left.+C_{b}\left(\gamma^{\sigma}-\frac{2 m_{t}}{\left(p_{1}^{\prime}+p_{2}^{\prime}\right)}\left(p_{1}^{\prime}+p_{2}^{\prime}\right)^{\sigma}\right)\right] \nu\left(k_{2}, s_{\bar{t}}\right) ; \\
M_{c}= & -i g_{s}^{2} T^{a} T^{b} \epsilon^{\mu}\left(p_{1}\right) \epsilon^{\nu}\left(p_{2}\right) \bar{u}\left(k_{1}, s_{t}\right)\left[A_{c} \gamma_{\mu}+B_{c}\left(k_{1}-p_{1}\right)_{\mu} \not p_{1}+C_{c}\left(k_{1}-p_{1}\right)_{\mu} \gamma^{5}\right. \\
& \left.+D_{c}\left(k_{1}-p_{1}\right)_{\mu} \not p_{1}^{\prime} \gamma^{5}+E_{c}\left(k_{1}-p_{1}\right)_{\mu}+F_{c} \gamma_{\mu} \gamma^{5}+G_{c} \not p_{1} \gamma_{\mu}+H_{c} \not \gamma_{1}^{\prime} \gamma_{\mu} \gamma^{5}\right] \\
& \frac{\left(k_{1}^{\prime}-\not p_{1}+m_{t}\right)}{\left(k_{1}-p_{1}\right)^{2}-m_{t}^{2} \gamma_{\nu} \nu\left(k_{2}, s_{\bar{t}}\right) ;} \\
& \\
M_{e}= & -i g_{s}^{2} T^{a} T^{b} \epsilon^{\mu}\left(p_{1}\right) \epsilon^{\nu}\left(p_{2}\right) \bar{u}\left(k_{1}, s_{t}\right) \gamma_{\mu} \frac{\left(k_{1}^{\prime}-\not p_{1}^{\prime}+m_{t}\right)}{\left(k_{1}-p_{1}\right)^{2}-m_{t}^{2}}\left[A_{e} \gamma_{\nu}+B_{e}\left(k_{1}-p_{1}\right)_{\nu} \not p_{2}\right. \\
& +C_{e}\left(k_{1}-p_{1}\right)_{\nu} \gamma^{5}+D_{e}\left(k_{1}-p_{1}\right)_{\nu} \not p_{2}^{\prime} \gamma^{5}+E_{e}\left(k_{1}-p_{1}\right)_{\nu}+F_{e} \gamma_{\nu} \gamma^{5} \\
& \left.+G_{e} \not p_{2}^{\prime} \gamma_{\mu}+H_{e} \not p_{2}^{\prime} \gamma_{\mu} \gamma^{5}\right] \nu\left(k_{2}, s_{\bar{t}}\right) ;
\end{aligned}
$$




$$
\begin{aligned}
M_{g}= & g_{s}^{2} T^{a} T^{b} \epsilon^{\mu}\left(p_{1}\right) \epsilon^{\nu}\left(p_{2}\right) \bar{u}\left(k_{1}, s_{t}\right) \gamma_{\mu} \frac{\not_{1}-\not p_{1}^{\prime}+m_{t}}{\left(k_{1}-p_{1}\right)^{2}-m_{t}^{2}}\left[\tilde{\Sigma}\left(k_{1}^{\prime}-\not p_{1}^{\prime}\right)+\left(k_{1}-\not p_{1}\right)\right. \\
& \left.\left(\delta Z_{V}-\delta Z_{A} \gamma_{5}\right)+m_{t} \delta m_{t}\right] \frac{\not p_{1}-p_{1}+m_{t}}{\left(k_{1}-p_{1}\right)^{2}-m_{t}^{2}} \gamma_{\nu} \nu\left(k_{2}, s_{\bar{t}}\right) ; \\
M_{i}=- & g_{s}^{2} \epsilon^{\mu}\left(p_{1}\right) \epsilon^{\nu}\left(p_{2}\right)\left[A_{i} g_{\mu \nu}+B_{i}\left(p_{2}-p_{1}\right)_{\mu}\left(p_{2}-p_{1}\right)_{\nu}+C_{i}\left(p_{2}-p_{1}\right)_{\mu} p_{1 \nu}\right. \\
+ & D_{i}\left(p_{2}-p_{1}\right)_{\mu}\left(p_{1}+p_{2}\right)_{\nu}+E_{i}\left(p_{1}+p_{2}\right)_{\mu}\left(p_{2}-p_{1}\right)_{\nu}+F_{i}\left(p_{1}+p_{2}\right)_{\mu} p_{1 \nu} \\
+ & G_{i}\left(p_{1}+p_{2}\right)_{\mu}\left(p_{1}+p_{2}\right)_{\nu}+H_{i}\left(p_{1}+p_{2}\right)^{\mu}\left(p_{1}+p_{2}\right)^{\nu} \varepsilon_{\mu \alpha \nu \sigma}+I_{i}\left(p_{1}+p_{2}\right)_{\mu} p_{1 \nu} \\
+ & \left.J_{i}\left(p_{1}+p_{2}\right)^{\alpha} p_{1}^{\sigma} \varepsilon_{\mu \alpha \nu \sigma}\right] \frac{1}{\left(p_{1}+p_{2}\right)^{2}-m_{t}^{2}} \bar{u}\left(k_{1}, s_{t}\right)\left(g_{V}+g_{A} \gamma_{5}\right) \nu\left(k_{2}, s_{\bar{t}}\right) .
\end{aligned}
$$

Here $g_{s}$ is the QCD coupling constant, $T^{i}$ stands for the color generator. $k_{1}\left(s_{t}\right)$ and $k_{2}\left(s_{\bar{t}}\right)$ denote the momentum(spin) of the top and anti-top quark, respectively.

For the renormalization of the ultraviolet divergences appearing in the evaluation of the vertex and fermion self-energy corrections, we have used the on-mass-shell renormalization scheme. The wave function renormalization constants can be determined from the top quark self-energy diagrams, which can be written as:

$$
\tilde{\Sigma}(\not \supset)=\not p\left[-\left(g_{A}^{2}+g_{V}^{2}\right) B_{1}\left(p^{2}\right)+2 g_{A} g_{V} B_{1}\left(p^{2}\right) \gamma_{5}\right]+m_{t}\left(g_{V}^{2}-g_{A}^{2}\right) B_{0}\left(p^{2}\right),
$$

with

$$
B_{1}=\frac{1}{2 p^{2}}\left[A_{0}\left(m_{t}^{2}\right)-A_{0}\left(M^{2}\right)-\left(m_{t}^{2}-M^{2}+p^{2}\right) B_{0}\right], \quad B_{0}=B_{0}\left(p^{2}, m_{t}^{2}, M^{2}\right) .
$$

Here $M$ denotes the mass of the scalar particle and $p$ is the momentum of the top quark t. $A_{0}$, and $B_{0}$ are the well-known one-point, two-point and three point scalar functions [15], which are given in terms of the Passarino-Veltman scalar functions. The expression forms of the form factors $A_{i}$ and $B_{i}$, etc have been given in the Appendix $A 1 \sim A 5$. In the on-shell scheme, the finite parts of the counter terms are determined by the requirement that the residue of the fermion propagator is equal to one, which fixes the wave function renormalization constraints $\delta Z_{V}, \delta Z_{A}$, and $\delta m_{t}$. Their expression forms are given in Appendix A6. The vector- and axis- vector coupling constants $g_{V}$ and $g_{A}$ can be written as: 
for the neutral top-pion $\pi_{t}^{0}, g_{V}=0, \quad g_{A}=(1-\varepsilon) m_{t} \sqrt{\nu_{w}-F_{t}^{2}} / \sqrt{2} F_{t} \nu_{w}$;

for the top-Higgs boson $h_{t}^{0}, g_{V}=-i(1-\varepsilon) m_{t} \sqrt{\nu_{w}-F_{t}^{2}} / \sqrt{2} F_{t} \nu_{w}, \quad g_{A}=0$.

The invariant amplitudes $M_{d}, M_{f}, M_{h}$ are same as $M_{c}, M_{e}, M_{g}$, respectively, but with $p_{1} \rightarrow p_{2}$. Because the expression form of $\mathcal{M}_{j}$ is lengthy, we do not present them here. Although, compared with the contributions for other diagrams, the contributions of Fig.1(j) to the $t \bar{t}$ production are small, our numerical results will include its contributions.

Similar as above, we can give the invariant amplitudes for the partonic processes $g g \rightarrow t \bar{t}$ and $q \bar{q} \rightarrow t \bar{t}$ contributed by the charged top-pions $\pi_{t}^{ \pm}$. In order not to make this paper too long, we do not present their explicit expressions here. However, in our calculation, we will include the contributions of the charged top-pions.

Using above amplitudes, it is straightforward to calculate the cross section $\hat{\sigma}^{i}(\hat{s})$ for the partonic process $i \rightarrow t \bar{t}$. The corresponding hadronic cross section $\sigma^{i}(\hat{s})$ can be obtained by folding $\hat{\sigma}^{i}(\hat{s})$ with the parton distribution functions (PDFs). The differentical cross section for the total hadronic cross section $\sigma(s)$ is given by:

$$
\frac{d \sigma(s)}{d X}=\sum_{i} \int d x_{1} d x_{2} f_{i / p}\left(x_{1}, \mu_{F}\right) f_{i / p}\left(x_{2}, \mu_{F}\right) \frac{d \hat{\sigma}^{i}(\hat{s})}{d X}
$$

where $i=q \bar{q}$ or $g g$ and $X$ can be chosen to be the $t \bar{t}$ invariant mass $M_{t \bar{t}}$ or the transverse momentum $P_{T}$ of the top quark. $\hat{s}=x_{1} x_{2} s$ is the effective center-of-mass (c.m.) energy squared for the partonic process $i \rightarrow t \bar{t}$. In our numerical calculation, we will use CTEQ6L $\mathrm{PDF}[16]$ for $f_{i / p}\left(x, \mu_{F}\right)$ and assume the factorization scale $\mu_{F}=m_{t}$.

The $t \bar{t}$ spin correlations manifest themselves in decay angular correlations, which are to be measured with respect to the chosen reference axes. If the $t(\bar{t})$ decays semileptonically $t \rightarrow b l^{+} \nu_{l}\left(\bar{t} \rightarrow \bar{b} l^{-} \nu_{l}\right)$, the charged lepton $l$ is the best spin analyzer [17]. A useful observable is the following double differential distribution $[4,5,6]$ :

$$
\frac{1}{\sigma} \frac{d \sigma^{2}}{d \cos \theta_{\ell^{+}} d \cos \theta_{\ell^{-}}}=\frac{1}{4}\left(1+B_{1} \cos \theta_{\ell^{+}}+B_{2} \cos \theta_{\ell^{-}}-C \cos \theta_{\ell^{+}} \cos \theta_{\ell^{-}}\right)
$$

Where $\sigma$ is the cross section of the process $p p \rightarrow t \bar{t} X \rightarrow l^{+} l^{-} X$ and $\theta_{\ell^{+}}\left(\theta_{\ell^{-}}\right)$expresses the angle between the $t(\bar{t})$ spin axis and the direction of flight of the lepton $l^{+}\left(l^{-}\right)$at the $t(\bar{t})$ rest frame. In this paper, we choose the helicity basis to analyze the $t \bar{t}$ spin correlations 
at the LHC. In this basis, the $t(\bar{t})$ spin axis is regarded as the direction of motion of the top (antitop) in the $t \bar{t}$ center-of-mass system. The coefficients $B_{1}$ and $B_{2}$ are associated with a polarization of the top and antitop quarks, and $C$ reflects the the strength of the $t \bar{t}$ spin correlations. In this paper we focus on investigating $C$, which can be expressed as:

$$
C=-k_{l^{+}} k_{l^{-}} A
$$

where $k_{l^{+}}$and $k_{l^{-}}$are the $t$ and $\bar{t}$ spin-analyzing powers and their values can be written as $k_{l^{+}}=-k_{l^{-}}=1$ at leading order. The parameter $A$ denotes the double spin asymmetry, which is defined as:

$$
A=4 \frac{\sigma(++)+\sigma(--)-\sigma(+-)-\sigma(-+)}{\sigma(++)+\sigma(--)+\sigma(+-)+\sigma(-+)}
$$

where $\sigma(+-)$ denotes the cross section for $\cos \theta_{l^{+}}>0$ and $\cos \theta_{l^{-}}<0$, etc.

The total matrix element squared for the process $p p \rightarrow t \bar{t}+X \rightarrow l^{+} l^{-}+X$ is given by:

$$
|M|^{2} \propto \operatorname{Tr}\left[\rho^{l^{+}} R^{i} \bar{\rho}^{l^{-}}\right]=\rho_{\alpha^{\prime} \alpha}^{l^{+}} R_{\alpha \beta, \alpha^{\prime} \beta^{\prime}}^{i} \bar{\rho}_{\beta^{\prime} \beta}^{l^{-}}
$$

in the narrow-width approximation for the top quark, where $\alpha^{\prime} \alpha$ and $\beta^{\prime} \beta$ are the spin labels of the $t$ and $\bar{t}$ quarks, respectively. The matrices $\rho^{l^{+}}$and $\bar{\rho}^{l^{-}}$are the density matrices corresponding to the decays $t \rightarrow l^{+}$and $\bar{t} \rightarrow l^{-}$, respectively. They can be written as:

$$
\begin{aligned}
& \rho_{\alpha^{\prime} \alpha}^{l^{+}}=M\left(t_{\alpha} \rightarrow b l^{+} \nu_{l}\right) M^{*}\left(t_{\alpha^{\prime}} \rightarrow b l^{+} \nu_{l}\right), \\
& \rho_{\beta^{\prime} \beta}^{l^{-}}=M\left(\bar{t}_{\beta} \rightarrow \bar{b} l^{-} \bar{\nu}_{l}\right) M^{*}\left(\bar{t}_{\beta^{\prime}} \rightarrow \bar{b} l^{-} \bar{\nu}_{l}\right) .
\end{aligned}
$$

$R_{\alpha \beta, \alpha^{\prime} \beta^{\prime}}^{i}$ is the $t \bar{t}$ production density matrice through the process $i$ in Eq.(3):

$$
R_{\alpha \beta, \alpha^{\prime} \beta^{\prime}}^{i}=\sum_{\text {initial spin }} M\left(i \rightarrow t_{\alpha} \bar{t}_{\beta}\right) M^{*}\left(i \rightarrow t_{\alpha^{\prime}} \bar{t}_{\beta^{\prime}}\right)
$$

where $M\left(i \rightarrow t_{\alpha} \bar{t}_{\beta}\right)$ are the invariant amplitudes given in Eqs.(5)-(10).

In the following section, we will use above formula to calculate some measurable quantities related to the $t \bar{t}$ production at the LHC. 


\section{The numerical results}

In our numerical estimation, we will take $m_{t}=172.7 \mathrm{GeV}, m_{b}=4.5 \mathrm{GeV}$, and $\alpha_{s}=0.1074$ [18]. Except for these SM input parameters, the contributions of the scalars predicted by the TC2 model to the $t \bar{t}$ production cross section are dependent on the free parameters $\varepsilon$, the masses of the top-pions and top-Higgs. The free parameter $\varepsilon$ parameterizes the portion of the extended technicolor contribution to the top quark mass. Numerical analysis shows that, with reasonable choice of other input parameters, $\varepsilon$ with order $10^{-2} \sim 10^{-1}$ may induce top-pions as massive as the top quark [10]. Precise value of $\varepsilon$ may be obtained by elaborately measuring the coupling strength between top-pion/topHiggs and top quarks at the next generation linear colliders. From the theoretical point of view, $\varepsilon$ with value from 0.01 to 0.1 is favored. In this paper, we will assume that its value is in the rang of $0.03 \sim 0.1$. Since the mass splitting between neutral and charged top-pions is very small, we assume $m_{\pi_{t}^{0}}=m_{\pi_{t}^{ \pm}}$. The top-pion mass is model-dependent and is usually of a few hundred $\mathrm{GeV}[2]$. About the top-Higgs mass, Ref. [13] gives a lower bound of about $2 m_{t}$, but it is an approximate analysis and the mass below $t \bar{t}$ threshold is also possible [19]. On the experimental side, the current experiments have restricted

the masses of the charged top-pions. For example, the absence of $t \rightarrow \pi_{t}^{+} b$ implies that $\pi_{t}^{+}>165 \mathrm{GeV}[20]$ and $R_{b}$ analysis yields $m_{\pi_{t}^{+}}>220 \mathrm{GeV}[21]$. For the masses of neutral top-pion and top-Higgs, the experimental restrictions on them are rather weak. So, in our numerical estimation, we will take $m_{\pi_{t}^{0}}=m_{\pi_{t}^{ \pm}}=m_{h_{t}^{0}}=M$ and assume that the value of $M$ is in the range of $200 \mathrm{GeV} \sim 450 \mathrm{GeV}$.

To see whether the effects of these new scalar particles on the top quark pair production can be detected via measuring observables at the LHC, we define the relative correction parameters as:

$$
\begin{aligned}
R_{1}= & \left|\frac{\sigma_{t o t}-\sigma_{S M}}{\sigma_{S M}}\right|, \quad R_{2}=\left|\frac{A_{t o t}-A_{S M}}{A_{S M}}\right|, \\
& R_{3}\left(P_{T}\right)=\left|\frac{d \sigma_{t o t} / d p_{T}-d \sigma_{S M} / d p_{T}}{d \sigma_{S M} / d p_{T}}\right| \\
& R_{4}\left(M_{t \bar{t}}\right)=\left|\frac{d \sigma_{t o t} / d M_{t \bar{t}}-d \sigma_{S M} / d M_{t \bar{t}}}{d \sigma_{S M} / d M_{t \bar{t}}}\right|
\end{aligned}
$$


Here the total $t \bar{t}$ production cross section $\sigma_{\text {tot }}$ includes the contributions coming from both the SM and the new scalars predicted by the TC2 model. $P_{T}$ and $M_{t \bar{t}}$ represent the top quark transverse momentum and the $t \bar{t}$ invariant mass, respectively.

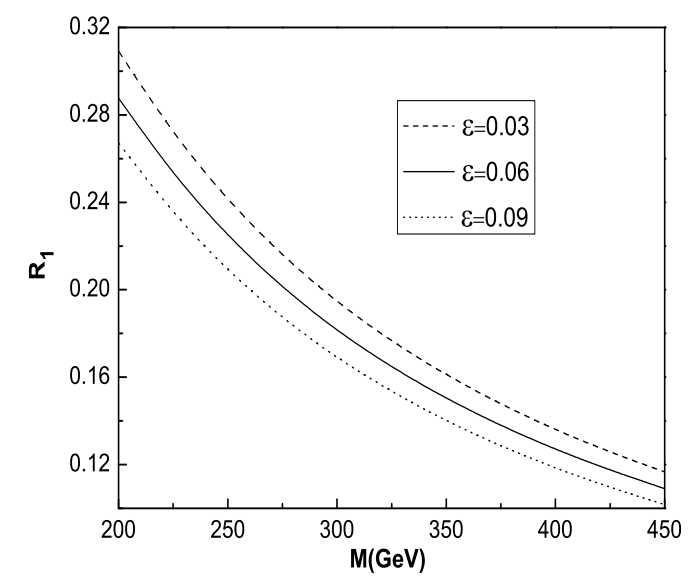

(a)

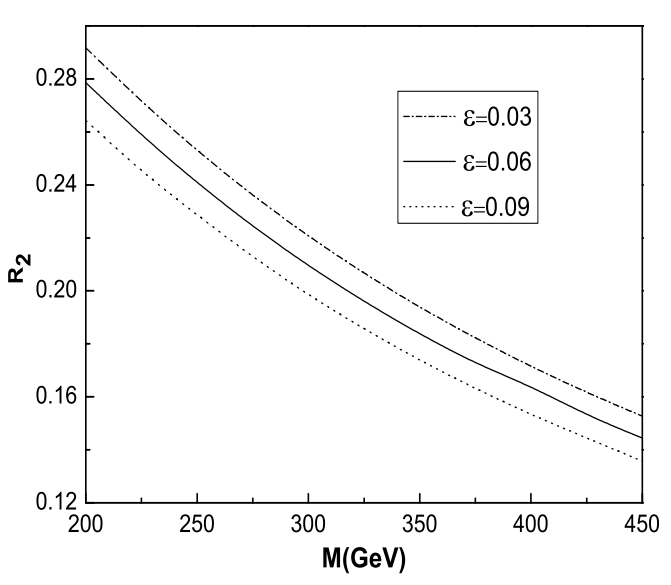

(b)

Figure 3: The relative correction parameters $R_{1}$ and $R_{2}$ are plotted as functions of the mass parameter $M$ for three values of the parameter $\varepsilon$.

Our numerical results show that, in the case of $m_{\pi_{t}^{0}}=m_{\pi_{t}^{ \pm}}=m_{h_{t}^{0}}$, the contributions of the neutral top-pion $\pi_{t}^{0}$ to the relative correction parameters $R_{i}$ are at the same order with those for the top-Higgs $h_{t}^{0}$. However, the contributions of the charged top-pions $\pi_{t}^{ \pm}$ are smaller than those for $\pi_{t}^{0}$ or $h_{t}^{0}$ at least by one order of magnitude. This is because, for $\pi_{t}^{ \pm}$, the fermion in each loop is bottom quark and furthermore there is no the s-channel Feynman diagram Fig.1(i). Summing up all of these contributions, we can obtain the contributions of the scalars predicted by the TC2 model to $R_{i}$, as shown in Fig.3 and Fig.4, in which we have taken the c.m. energy $\sqrt{s}=14 T e V$. From these figures one can see that these scalar particles can indeed produce significant corrections to the observables, which are related the top quark pair production at the LHC. The correction effects decrease as the mass parameter $M$ and the parameter $\varepsilon$ increasing. In wide range of the parameter space, the correction effects of the new scalars to the $t \bar{t}$ production cross section $\sigma(t \bar{t})$ are significant large, and the value of the relative correction parameter $R_{1}$ is larger than 


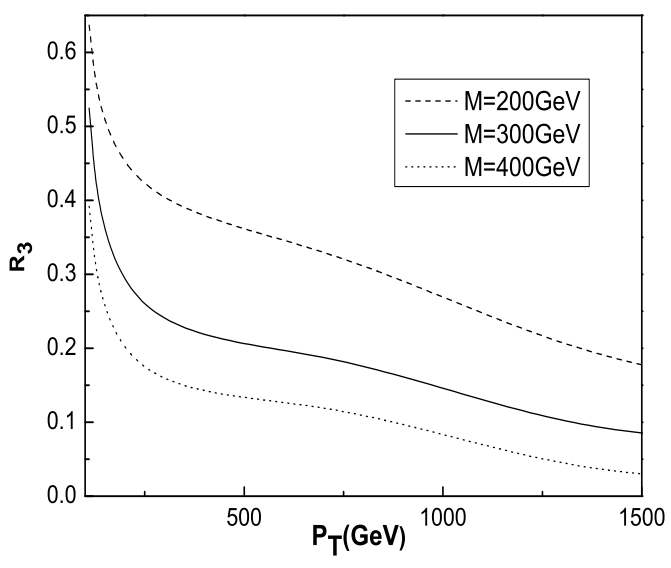

(a)

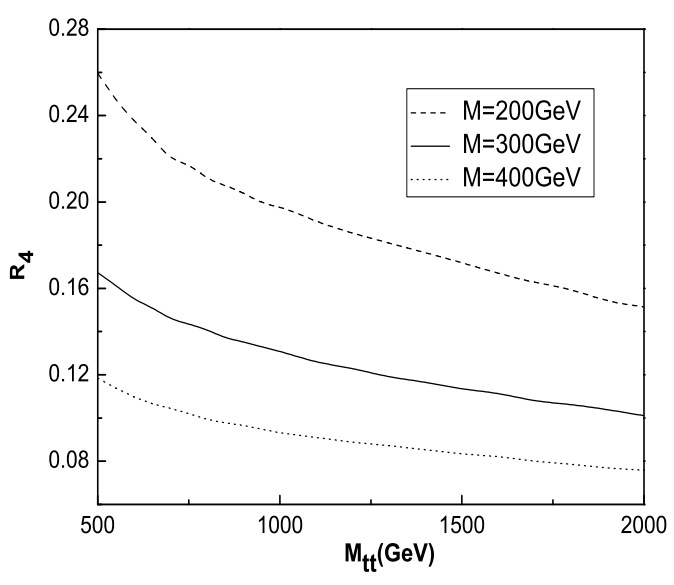

(b)

Figure 4: The relative correction parameter $R_{3}\left(R_{4}\right)$ as a function of the transverse momentum $P_{T}\left(\right.$ invariant mass $\left.M_{t t}\right)$ for $\varepsilon=0.05$ and three values of the mass parameter $M$.

10\%, which might be detected at the LHC. For the masses of the scalars equaling to $300 \mathrm{GeV}$ and $\varepsilon=0.05$, the values of the relative correction parameters $R_{3}$ and $R_{4}$ are in the ranges of $9 \% \sim 52 \%$ and $10 \% \sim 17 \%$, respectively. For $200 \mathrm{GeV} \leq M \leq 450 \mathrm{GeV}$ and $0.03 \leq \varepsilon \leq 0.09$, the values of the parameter $R_{2}$ is in the range of $14 \% \sim 29 \%$. Ref. [22] has shown that the spin asymmetry $A$ of the top-antitop pairs in the SM will be measured with a precision of about $6 \%$ after one LHC year at low luminosity $\left(10 f b^{-1}\right)$. Thus, the correction effects of the scalars to the spin asymmetry $A$ should be detected.

Certainly, the scalar particles predicted by the TC2 models can also produce correction effects on the observables, which are related the top quark pair production at the Tevatron. However, since the partonic process $q \bar{q} \rightarrow t \bar{t}$ is the dominate process at the Tevatron, the contributions of these new particles to the correlative observables are smaller than those for the LHC. For instance, in wide range of the parameter space of the TC2 model, the relative correction value of these new scalars to the $t \bar{t}$ production cross section $\sigma(t \bar{t})$ is in the range of $3 \% \sim 11 \%$ at the Tevatron.

In conclusion, we have considered the contributions of the new scalars predicted by the 
TC2 model to the $t \bar{t}$ production and the $t \bar{t}$ spin correlations at the LHC. Our numerical results show that these new particles can generate significant corrections to some correlative observables. The LHC might detect these correction effects in near future. Thus, one can use the process $p p \rightarrow t \bar{t}+X$ to test the possible signatures of these new scalars at the LHC. Furthermore, most of the new physics models in the topcolor scenario predict the existence of the neutral and charged scalars, which have similar features as those for the TC2 model. So our conclusions are apply to the topcolor scenario.

\section{Acknowledgments}

J. Y. Liu thanks Zong-Guo Si for helpful discussions. This work is supported in part by the National Natural Science Foundation of China under Grant No.10975067, the Specialized Research Fund for the Doctoral Program of Higher Education(SRFDP) (No.200801650002), the Natural Science Foundation of Liaoning Science Committee(No.2008 2148), and the Foundation of Liaoning Educational Committee(No.2007T086). 


\section{Appendix :}

In this Appendix, we list the form factors of the invariant amplitudes for the partonic processes $g\left(p_{1}\right) g\left(p_{2}\right) \rightarrow t\left(k_{1}, s_{t}\right) \bar{t}\left(k_{2}, s_{\bar{t}}\right)$ and $q\left(p_{1}^{\prime}\right) \bar{q}\left(p_{2}^{\prime}\right) \rightarrow t\left(k_{1}, s_{t}\right) \bar{t}\left(k_{2}, s_{\bar{t}}\right)$. The counterterms $\delta Z_{V}, \delta Z_{A}$, and $\delta m_{t}$ coming from the top quark self-energy contributions are listed in Appendix A6.

\section{A1. Form factors appearing in $M_{a}$}

$$
\begin{aligned}
A_{a}= & 1+\frac{1}{16 \pi^{2}} \frac{g_{A}^{2}+g_{V}^{2}}{2 m_{t}^{2}}\left[A_{0}\left(M^{2}\right)-A_{0}\left(m_{t}^{2}\right)+\left(2 m_{t}^{2}-M^{2}\right)\right] B_{0}\left(m_{t}^{2}, M^{2}, m_{t}^{2}\right)+\frac{1}{16 \pi^{2}} \\
& {\left[\left(g_{A}^{2}+g_{V}^{2}\right)\left(M^{2}-2 m_{t}^{2}\right)-\left(g_{A}^{2}-g_{V}^{2}\right) 2 m_{t}^{2}\right] B_{0}^{\prime}\left(m_{t}^{2}\right)+\left\{-\frac{g_{V} g_{V}^{*}}{16 \pi^{2}}\left\{\frac{\hat{s}}{2\left(\hat{s}-4 m_{t}^{2}\right)}\right.\right.} \\
& +\frac{2}{\hat{s}-4 m_{t}^{2}}\left[A_{0}\left(m_{t}^{2}\right)-A_{0}\left(M^{2}\right)\right]+B_{0}\left(\hat{s}^{2}, m_{t}^{2}, m_{t}^{2}\right) \frac{1}{2\left(\hat{s}-4 m_{t}^{2}\right)^{2}}\left[-48 m_{t}^{4}+\left(-16 m_{t}^{2}\right.\right. \\
& \left.\left.+16 M^{2}+14 \hat{s}\right) m_{t}^{2}+8 m_{t}^{2} \hat{s}-\hat{s}^{2}-2 m_{t}^{2} \hat{s}+2 M^{2} \hat{s}\right]+\frac{1}{2\left(\hat{s}-4 m_{t}^{2}\right)^{2}} B_{0}\left(m_{t}^{2}, M^{2}, m_{t}^{2}\right) \\
& {\left[32 m_{t}^{4}+\left(32 m_{t}^{2}-32 M^{2}-6 \hat{s}^{2}\right) m_{t}^{2}-8 m_{t}^{2} \hat{s}-2 \hat{s}\left(m_{t}^{2}-M^{2}\right)\right]+\frac{1}{2\left(\hat{s}-4 m_{t}^{2}\right)^{2}} } \\
& C_{0}\left(0, m_{t}^{2}, p_{1} p_{2 s}, m_{t}^{2}, m_{t}^{2}, M^{2}\right)\left[48 m_{t}^{6}+\left(32 m_{t}^{2}-32 M^{2}-6 \hat{s}\right) m_{t}^{4}+\left(32 m_{t}^{3}\right.\right. \\
& \left.-32 m_{t} M^{2}-24 m_{t} \hat{s}\right) m_{t}^{3}+\left(16 m_{t}^{4}+20 M^{2} \hat{s}+16 M^{4}-28 m_{t}^{2} \hat{s}+2 \hat{s}^{2}-32 m_{t}^{2} M^{2}\right) m_{t}^{2} \\
& \left.\left.+\left(4 m_{t} \hat{s}^{2}-8 m_{t}^{3} \hat{s}+8 m_{t} M^{2} \hat{s}\right) m_{t}+2 m_{t}^{2} \hat{s}+2 m_{t}^{4} \hat{s}+2 M^{4} \hat{s}-4 m_{t}^{2} M^{2} \hat{s}\right]\right\}-\frac{g_{A} g_{A}^{*}}{16 \pi^{2}} \\
& \left\{\frac{\hat{s}}{2\left(\hat{s}-4 m_{t}^{2}\right)}+\frac{2}{\hat{s}-4 m_{t}^{2}}\left[A_{0}\left(m_{t}^{2}\right)-A_{0}\left(M^{2}\right)\right]+\frac{1}{2\left(\hat{s}-4 m_{t}^{2}\right)^{2}} B_{0}\left(\hat{s}^{2}, m_{t}^{2}, m_{t}^{2}\right)\right. \\
& {\left[16 m_{t}^{4}+\left(16 M^{2}-16 m_{t}^{2}+14 \hat{s}\right) m_{t}^{2}-8 m_{t}^{2} \hat{s}-\hat{s}^{2}-2 m_{t}^{2} \hat{s}+2 M^{2} \hat{s}\right] } \\
& \frac{1}{2\left(\hat{s}-4 m_{t}^{2}\right)^{2}} B_{0}\left(m_{t}^{2}, M^{2}, m_{t}^{2}\right)\left[-32 m_{t}^{4}+\left(32 m_{t}^{2}-32 M^{2}-6 \hat{s}^{2}\right) m_{t}^{2}+8 m_{t}^{2} \hat{s}\right. \\
& \left.-2 \hat{s}\left(m_{t}^{2}-M^{2}\right)\right]+\frac{1}{2\left(\hat{s}-4 m_{t}^{2}\right)^{2}} C_{0}\left(0, m_{t}^{2}, p_{1} p_{2 s}, m_{t}^{2}, m_{t}^{2}, M^{2}\right)\left[48 m_{t}^{6}\right. \\
& +\left(32 m_{t}^{2}-32 M^{2}-6 \hat{s}\right) m_{t}^{4}+\left(32 m_{t}^{3}+32 m_{t} M^{2}-24 m_{t} \hat{s}\right) m_{t}^{3} \\
& +\left(16 m_{t}^{4}+20 M^{2} \hat{s}+16 M^{4}-28 m_{t}^{2} \hat{s}+2 \hat{s}^{2}-32 m_{t}^{2} M^{2}\right) m_{t}^{2}+\left(4 m_{t} \hat{s}^{2}-8 m_{t}^{3} \hat{s}\right. \\
& \left.\left.\left.\left.+8 m_{t} M^{2} \hat{s}\right) m_{t}+2 m_{t}^{2} \hat{s}+2 m_{t}^{4} \hat{s}+2 M^{4} \hat{s}-4 m_{t}^{2} M^{2} \hat{s}\right]\right\}\right\}, \\
&
\end{aligned}
$$




$$
\begin{aligned}
& B_{a}=\frac{g_{V} g_{V}^{*}}{16 \pi^{2}}\left\{\frac{m_{t}}{\hat{s}-4 m_{t}^{2}}+\frac{1}{m_{t}\left(\hat{s}-4 m_{t}^{2}\right)}\left[A_{0}\left(m_{t}^{2}\right)-A_{0}\left(M^{2}\right)\right]+B_{0}\left(\hat{s}, m_{t}^{2}, m_{t}^{2}\right) \frac{1}{\left(\hat{s}-4 m_{t}^{2}\right)^{2}}\right. \\
& {\left[2 m_{t}^{3}-8 m_{t}^{3}+\left(-6 m_{t}^{2}+6 M^{2}+\hat{s}\right) m_{t}+2 m_{t} \hat{s}\right]+B_{0}\left(m_{t}^{2}, M^{2}, m_{t}^{2},\right) \frac{1}{m_{t}\left(\hat{s}-4 m_{t}^{2}\right)^{2}}} \\
& {\left[6 m_{t}^{4}+\left(10 m_{t}^{2}-10 M^{2}-\hat{s}\right) m_{t}^{2}-2 m_{t}^{2} \hat{s}+\left(M^{2}-m_{t}^{2}\right) \hat{s}\right]} \\
& +C_{0}\left(0, m_{t}^{2}, p_{1} p_{2 s}, m_{t}^{2}, m_{t}^{2}, M^{2}\right) \frac{(-2)}{\left(\hat{s}-4 m_{t}^{2}\right)^{2}}\left[5 m_{t}^{5}+\left(2 m_{t}^{2}+2 M^{2}-\hat{s}\right) m_{t}^{3}\right. \\
& \left.-\left(4 m_{t}^{2}-4 M^{2}+\hat{s}\right) m_{t}^{3}\right]+\left[-3 m_{t}^{4}+m_{t}^{2}\left(6 M^{2}+\hat{s}\right)-3 M^{4}-2 M^{2} \hat{s}\right] m_{t} \\
& \left.+m_{t}\left(m_{t}^{2}-M^{2}\right) \hat{s}\right\}+\frac{g_{A} g_{A}^{*}}{16 \pi^{2}}\left\{\frac{m_{t}}{\hat{s}-4 m_{t}^{2}}+\frac{1}{m_{t}\left(\hat{s}-4 m_{t}^{2}\right)}\left[A_{0}\left(m_{t}^{2}\right)-A_{0}\left(M^{2}\right)\right]\right. \\
& +B_{0}\left(\hat{s}, m_{t}^{2}, m_{t}^{2}\right) \frac{1}{\left(\hat{s}-4 m_{t}^{2}\right)^{2}}\left[2 m_{t}^{3}+8 m_{t}^{3}+\left(-6 m_{t}^{2}+6 M^{2}+\hat{s}\right) m_{t}-2 m_{t} \hat{s}\right] \\
& +B_{0}\left(m_{t}^{2}, M^{2}, m_{t}^{2},\right) \frac{1}{m_{t}\left(\hat{s}-4 m_{t}^{2}\right)^{2}}\left[-10 m_{t}^{4}+\left(10 m_{t}^{2}-10 M^{2}-\hat{s}\right) m_{t}^{2}+2 m_{t}^{2} \hat{s}\right. \\
& \left.+\left(M^{2}-m_{t}^{2}\right) \hat{s}\right]+C_{0}\left(0, m_{t}^{2}, p_{1} p_{2 s}, m_{t}^{2}, m_{t}^{2}, M^{2}\right) \frac{(-2)}{\left(\hat{s}-4 m_{t}^{2}\right)^{2}}\left[-3 m_{t}^{5}+\left(2 m_{t}^{2}+2 M^{2}\right.\right. \\
& \left.-\hat{s}) m_{t}^{3}-\left(4 m_{t}^{2}-4 M^{2}+\hat{s}\right) m_{t}^{3}\right]+\left[-3 m_{t}^{4}+m_{t}^{2}\left(6 M^{2}+\hat{s}\right)-3 M^{4}-2 M^{2} \hat{s}\right] m_{t} \\
& \left.\left.-m_{t}\left(m_{t}^{2}-M^{2}\right) \hat{s}\right\}\right\} \text {, } \\
& C_{a}=-\frac{g_{V} g_{A}^{*}}{16 \pi^{2}}\left\{-1+\frac{1}{\hat{s}-4 m_{t}^{2}} B_{0}\left(\hat{s}, m_{t}^{2}, m_{t}^{2}\right)\left(4 m_{t}^{2}-2 M^{2}+\hat{s}\right)+B_{0}\left(m_{t}^{2}, M^{2}, m_{t}^{2}\right)\right. \\
& \left(\frac{-2}{\hat{s}-4 m_{t}^{2}}\right)\left(4 m_{t}^{2}+M^{2}\right)+C_{0}\left(0, m_{t}^{2}, p_{1} p_{2 s}, m_{t}^{2}, m_{t}^{2}, M^{2}\right)\left(\frac{-2}{\hat{s}-4 m_{t}^{2}}\right)\left(4 m_{t}^{2}+M^{2}\right) \\
& \left.\left[2 m_{t}^{4}-\left(2 m_{t}^{2}+2 M^{2}+\hat{s}\right) m_{t}^{2}+M^{4}-2 m_{t}^{2} M^{2}+m_{t}^{2} \hat{s}\right]\right\} \text {. }
\end{aligned}
$$

Here $\hat{s}=\left(p_{1}+p_{2}\right)^{2} . C_{0}$ is the three point scalar function [15]. We also introduce the following shorthhand notation:

$$
\left.B_{0}^{\prime}\left(m_{t}^{2}\right) \equiv \frac{\partial}{\partial P^{2}} B_{0}\left(m_{t}^{2} ; M^{2}, m_{t}^{2}\right)\right|_{p^{2}=m_{t}^{2}}
$$




\section{A2. Form factors appearing in $M_{b}$}

$$
\begin{aligned}
& A_{b}=\frac{g_{A}^{2}+g_{V}^{2}}{32 \pi^{2} m_{t}^{2}}\left[A_{0}\left(M^{2}\right)-B_{0}\left(m_{t}^{2}, M^{2}, m_{t}^{2}\right) A_{0}\left(m_{t}^{2}\right)+\left(2 m_{t}^{2}-M^{2}\right)\right]+\frac{1}{16 \pi^{2}}\left[\left(g_{A}^{2}+g_{V}^{2}\right)\right. \\
& \left.\left(M^{2}-2 m_{t}^{2}\right)-\left(g_{A}^{2}-g_{V}^{2}\right) 2 m_{t}^{2}\right] B_{0}^{\prime}\left(m_{t}^{2}\right)+\left\{-\frac{g_{V} g_{V}^{*}}{16 \pi^{2}}\left\{\frac{\hat{s}}{2\left(\hat{s}-4 m_{t}^{2}\right)}+\frac{2}{\hat{s}-4 m_{t}^{2}}\right.\right. \\
& {\left[A_{0}\left(m_{t}^{2}\right)-A_{0}\left(M^{2}\right)\right]+\frac{1}{2\left(\hat{s}-4 m_{t}^{2}\right)^{2}} B_{0}\left(\hat{s}^{2}, m_{t}^{2}, m_{t}^{2}\right)\left[-48 m_{t}^{4}+\left(-16 m_{t}^{2}\right.\right.} \\
& \left.\left.+16 M^{2}+14 \hat{s}\right) m_{t}^{2}+8 m_{t}^{2} \hat{s}-\hat{s}^{2}-2 m_{t}^{2} \hat{s}+2 M^{2} \hat{s}\right]+\frac{1}{2\left(\hat{s}-4 m_{t}^{2}\right)^{2}} B_{0}\left(m_{t}^{2}, M^{2}, m_{t}^{2}\right) \\
& {\left[32 m_{t}^{4}+\left(32 m_{t}^{2}-32 M^{2}-6 \hat{s}^{2}\right) m_{t}^{2}-8 m_{t}^{2} \hat{s}-2 \hat{s}\left(m_{t}^{2}-M^{2}\right)\right]+\frac{1}{2\left(\hat{s}-4 m_{t}^{2}\right)^{2}}} \\
& C_{0}\left(0, m_{t}^{2}, p_{1} p_{2 s}, m_{t}^{2}, m_{t}^{2}, M^{2}\right)\left[48 m_{t}^{6}+\left(32 m_{t}^{2}-32 M^{2}-6 \hat{s}\right) m_{t}^{4}+\left(32 m_{t}^{3}\right.\right. \\
& \left.-32 m_{t} M^{2}-24 m_{t} \hat{s}\right) m_{t}^{3}+\left(16 m_{t}^{4}+20 M^{2} \hat{s}+16 M^{4}-28 m_{t}^{2} \hat{s}+2 \hat{s}^{2}-32 m_{t}^{2} M^{2}\right) m_{t}^{2} \\
& \left.\left.+\left(4 m_{t} \hat{s}^{2}-8 m_{t}^{3} \hat{s}+8 m_{t} M^{2} \hat{s}\right) m_{t}+2 m_{t}^{2} \hat{s}+2 m_{t}^{4} \hat{s}+2 M^{4} \hat{s}-4 m_{t}^{2} M^{2} \hat{s}\right]\right\}-\frac{g_{A} g_{A}^{*}}{16 \pi^{2}} \\
& \left\{\frac{\hat{s}}{2\left(\hat{s}-4 m_{t}^{2}\right)}+\frac{2}{\hat{s}-4 m_{t}^{2}}\left[A_{0}\left(m_{t}^{2}\right)-A_{0}\left(M^{2}\right)\right]+\frac{1}{2\left(\hat{s}-4 m_{t}^{2}\right)^{2}} B_{0}\left(\hat{s}^{2}, m_{t}^{2}, m_{t}^{2}\right)\right. \\
& {\left[16 m_{t}^{4}+\left(16 M^{2}-16 m_{t}^{2}+14 \hat{s}\right) m_{t}^{2}-8 m_{t}^{2} \hat{s}-\hat{s}^{2}-2 m_{t}^{2} \hat{s}+2 M^{2} \hat{s}\right] \frac{1}{2\left(\hat{s}-4 m_{t}^{2}\right)^{2}}} \\
& B_{0}\left(m_{t}^{2}, M^{2}, m_{t}^{2}\right)\left[-32 m_{t}^{4}+\left(32 m_{t}^{2}-32 M^{2}-6 \hat{s}^{2}\right) m_{t}^{2}+8 m_{t}^{2} \hat{s}-2 \hat{s}\left(m_{t}^{2}-M^{2}\right)\right] \\
& +\frac{1}{2\left(\hat{s}-4 m_{t}^{2}\right)^{2}} C_{0}\left(0, m_{t}^{2}, p_{1} p_{2 s}, m_{t}^{2}, m_{t}^{2}, M^{2}\right)\left[48 m_{t}^{6}+\left(32 m_{t}^{2}-32 M^{2}-6 \hat{s}\right) m_{t}^{4}\right. \\
& +\left(32 m_{t}^{3}+32 m_{t} M^{2}-24 m_{t} \hat{s}\right) m_{t}^{3}+\left(16 m_{t}^{4}+20 M^{2} \hat{s}+16 M^{4}-28 m_{t}^{2} \hat{s}+2 \hat{s}^{2}\right. \\
& \left.-32 m_{t}^{2} M^{2}\right) m_{t}^{2}+\left(4 m_{t} \hat{s}^{2}-8 m_{t}^{3} \hat{s}+8 m_{t} M^{2} \hat{s}\right) m_{t}+2 m_{t}^{2} \hat{s}+2 m_{t}^{4} \hat{s} \\
& \left.\left.\left.+2 M^{4} \hat{s}-4 m_{t}^{2} M^{2} \hat{s}\right]\right\}+1\right\} \\
& B_{b}=\frac{g_{V} g_{V}^{*}}{16 \pi^{2}}\left\{\frac{m_{t}}{\hat{s}-4 m_{t}^{2}}+\frac{1}{m_{t}\left(\hat{s}-4 m_{t}^{2}\right)}\left[A_{0}\left(m_{t}^{2}\right)-A_{0}\left(M^{2}\right)\right]+\frac{1}{\left(\hat{s}-4 m_{t}^{2}\right)^{2}} B_{0}\left(\hat{s}, m_{t}^{2}, m_{t}^{2}\right)\right. \\
& {\left[2 m_{t}^{3}-8 m_{t}^{3}+\left(-6 m_{t}^{2}+6 M^{2}+\hat{s}\right) m_{t}+2 m_{t} \hat{s}\right]+B_{0}\left(m_{t}^{2}, M^{2}, m_{t}^{2},\right) \frac{1}{m_{t}\left(\hat{s}-4 m_{t}^{2}\right)^{2}}} \\
& {\left[6 m_{t}^{4}+\left(10 m_{t}^{2}-10 M^{2}-\hat{s}\right) m_{t}^{2}-2 m_{t}^{2} \hat{s}+\left(M^{2}-m_{t}^{2}\right) \hat{s}\right]}
\end{aligned}
$$




$$
\begin{aligned}
- & \frac{2}{\left(\hat{s}-4 m_{t}^{2}\right)^{2}} C_{0}\left(0, m_{t}^{2}, p_{1} p_{2 s}, m_{t}^{2}, m_{t}^{2}, M^{2}\right)\left[5 m_{t}^{5}+\left(2 m_{t}^{2}+2 M^{2}-\hat{s}\right) m_{t}^{3}\right. \\
- & \left.\left(4 m_{t}^{2}-4 M^{2}+\hat{s}\right) m_{t}^{3}\right]+\left[-3 m_{t}^{4}+m_{t}^{2}\left(6 M^{2}+\hat{s}\right)-3 M^{4}-2 M^{2} \hat{s}\right] m_{t} \\
+ & \left.m_{t}\left(m_{t}^{2}-M^{2}\right) \hat{s}\right\}+\frac{g_{A} g_{A}^{*}}{16 \pi^{2}}\left\{\frac{m_{t}}{\hat{s}-4 m_{t}^{2}}+\frac{1}{m_{t}\left(\hat{s}-4 m_{t}^{2}\right)}\left[A_{0}\left(m_{t}^{2}\right)-A_{0}\left(M^{2}\right)\right]\right. \\
+ & B_{0}\left(\hat{s}, m_{t}^{2}, m_{t}^{2}\right) \frac{1}{\left(\hat{s}-4 m_{t}^{2}\right)^{2}}\left[2 m_{t}^{3}+8 m_{t}^{3}+\left(-6 m_{t}^{2}+6 M^{2}+\hat{s}\right) m_{t}-2 m_{t} \hat{s}\right] \\
+ & B_{0}\left(m_{t}^{2}, M^{2}, m_{t}^{2},\right) \frac{1}{m_{t}\left(\hat{s}-4 m_{t}^{2}\right)^{2}}\left[-10 m_{t}^{4}+\left(10 m_{t}^{2}-10 M^{2}-\hat{s}\right) m_{t}^{2}+2 m_{t}^{2} \hat{s}\right. \\
+ & \left.\left(M^{2}-m_{t}^{2}\right) \hat{s}\right]-C_{0}\left(0, m_{t}^{2}, p_{1} p_{2 s}, m_{t}^{2}, m_{t}^{2}, M^{2}\right) \frac{2}{\left(\hat{s}-4 m_{t}^{2}\right)^{2}}\left[-3 m_{t}^{5}+\left(2 m_{t}^{2}+2 M^{2}\right.\right. \\
- & \left.\hat{s}) m_{t}^{3}-\left(4 m_{t}^{2}-4 M^{2}+\hat{s}\right) m_{t}^{3}\right]+\left[-3 m_{t}^{4}+m_{t}^{2}\left(6 M^{2}+\hat{s}\right)-3 M^{4}-2 M^{2} \hat{s}\right] m_{t} \\
- & \left.\left.m_{t}\left(m_{t}^{2}-M^{2}\right) \hat{s}\right\}\right\}, \\
& \quad-\frac{g_{V} g_{A}^{*}}{16 \pi^{2}}\left\{-1+\frac{1}{\hat{s}-4 m_{t}^{2}}\left(4 m_{t}^{2}-2 M^{2}+\hat{s}\right) B_{0}\left(\hat{s}, m_{t}^{2}, m_{t}^{2}\right)-B_{0}\left(m_{t}^{2}, M^{2}, m_{t}^{2}\right)\right. \\
& \quad\left(\frac{2}{\hat{s}-4 m_{t}^{2}}\right)\left(4 m_{t}^{2}+M^{2}\right)-\frac{2}{\hat{s}-4 m_{t}^{2}} C_{0}\left(0, m_{t}^{2}, p_{1} p_{2 s}, m_{t}^{2}, m_{t}^{2}, M^{2}\right)\left(4 m_{t}^{2}+M^{2}\right) \\
& {\left.\left[2 m_{t}^{4}-\left(2 m_{t}^{2}+2 M^{2}+\hat{s}\right) m_{t}^{2}+M^{4}-2 m_{t}^{2} M^{2}+m_{t}^{2} \hat{s}\right]\right\} . }
\end{aligned}
$$

\section{A3. Form factors appearing in $M_{c}$}

$$
\begin{aligned}
A_{c}= & \frac{1}{16 \pi^{2}} \frac{1}{4\left(p_{1}^{2}+p_{1} \cdot p_{2}\right)}\left\{\left(g_{A}^{2}+g_{V}^{2}\right)\left[\left(B_{0}\left(0, m_{t}^{2}, m_{t}^{2}\right)-B_{0}\left(p_{1} p_{2 s}, m_{t}^{2}, M^{2}\right)\right)\left(2 m_{t}^{2}-M^{2}\right)\right]\right. \\
& \left.+2\left(p_{1}^{2}+p_{1} \cdot p_{2}\right)\left(-1+B_{0}\left(p_{1} p_{2 s}, m_{t}^{2}, M^{2}\right)-4 C_{0}\left(0, m_{t}^{2}, p_{1} p_{2 s}, m_{t}^{2}, m_{t}^{2}, M^{2}\right) m_{t}^{2}\right)\right\} \\
& -\delta Z_{V}
\end{aligned}
$$

$$
\begin{aligned}
B_{c}= & -\frac{1}{16 \pi^{2}} \frac{1}{4\left[m_{t}^{2}-2\left(p_{2}^{2}+p_{2} \cdot p_{1}\right)\right]\left(p_{2}^{2}+p_{2} \cdot p_{1}\right)^{2}}\left(g_{A}^{2}+g_{V}^{2}\right)\left\{\left(B_{0}\left(0, m_{t}^{2}, m_{t}^{2}\right)\right.\right. \\
& \left.-B_{0}\left(p_{1} p_{2 s}, m_{t}^{2}, M^{2}\right)\right)\left(2 m_{t}^{2}-M^{2}\right) m_{t}^{2}-2\left[2 B_{0}\left(p_{1} p_{2 s}, m_{t}^{2}, M^{2}\right) m_{t}^{2}-B_{0}\left(m_{t}^{2}, m_{t}^{2}, M^{2}\right)\right. \\
& m_{t}^{2}-2 B_{0}\left(p_{1} p_{2 s}, m_{t}^{2}, M^{2}\right) M^{2}+2 B_{0}\left(m_{t}^{2}, m_{t}^{2}, M^{2}\right) M^{2}+m_{t}^{2}+B_{0}\left(p_{1} p_{2 s}, m_{t}^{2}, M^{2}\right) m_{t}^{2} \\
& \left.-B_{0}\left(m_{t}^{2}, m_{t}^{2}, M^{2}\right) m_{t}^{2}-2 C_{0}\left(0, m_{t}^{2}, p_{1} p_{2 s}, m_{t}^{2}, m_{t}^{2}, M^{2}\right) m_{t}^{4}+A_{0}\left(m_{t}^{2}\right)+A_{0}\left(M^{2}\right)\right] \\
& \left.\left(p_{1}^{2}+p_{1} \cdot p_{2}\right)+4\left(1+2 C_{0}\left(0, m_{t}^{2}, p_{1} p_{2 s}, m_{t}^{2}, m_{t}^{2}, M^{2}\right) m_{t}^{2}\right)\left(p_{2}^{2}+p_{2} \cdot p_{1}\right)^{2}\right\},
\end{aligned}
$$




$$
\begin{aligned}
C_{c}= & \frac{1}{16 \pi^{2}} \frac{1}{\left(p_{1}^{2}+p_{1} \cdot p_{2}\right)\left[m_{t}^{2}-2\left(p_{1}^{2}+p_{1} \cdot p_{2}\right)\right] m_{t}}\left\{\left(B_{0}\left(p_{1} p_{2 s}, m_{t}^{2}, M^{2}\right)-B_{0}\left(m_{t}^{2}, m_{t}^{2}, M^{2}\right)\right)\right. \\
& m_{t}^{2}\left(2 m_{t}^{2}-M^{2}\right) g_{A} g_{V}+2\left(B_{0}\left(m_{t}^{2}, m_{t}^{2}, M^{2}\right) m_{t}^{2}-B_{0}\left(m_{t}^{2}, m_{t}^{2}, M^{2}\right) M^{2}\right. \\
& \left.-B_{0}\left(p_{1} p_{2 s}, m_{t}^{2}, M^{2}\right) m_{t}^{2}+B_{0}\left(m_{t}^{2}, m_{t}^{2}, M^{2}\right) m_{t}^{2}-A_{0}\left(m_{t}^{2}\right)+A_{0} M^{2}\right) \\
& \left.\left.\left(p_{1}^{2}+p_{1} \cdot p_{2}\right)\right)\right\} \\
D_{c}= & \frac{1}{16 \pi^{2}} \frac{-1}{2\left[m_{t}^{2}-2\left(p_{2}^{2}+p_{2} \cdot p_{1}\right)\right]\left(p_{2}^{2}+p_{2} \cdot p_{1}\right)^{2}}\left[\left(B_{0}\left(0, m_{t}^{2}, m_{t}^{2}\right)-B_{0}\left(p_{1} p_{2 s}, m_{t}^{2}, M^{2}\right)\right)\right. \\
& m_{t}^{2}\left(2 m_{t}^{2}-M^{2}\right)-2\left(B_{0}\left(0, m_{t}^{2}, m_{t}^{2}\right) m_{t}^{2}-2 B_{0}\left(p_{1} p_{2 s}, m_{t}^{2}, M^{2}\right) m_{t}^{2}-B_{0}\left(0, m_{t}^{2}, m_{t}^{2}\right) M^{2}\right. \\
& +2 B_{0}\left(p_{1} p_{2 s}, m_{t}^{2}, M^{2}\right) M^{2}-m_{t}^{2}+B_{0}\left(0, m_{t}^{2}, m_{t}^{2}\right) m_{t}^{2}-B_{0}\left(p_{1} p_{2 s}, m_{t}^{2}, M^{2}\right) m_{t}^{2} \\
& \left.-2 C_{0}\left(0, m_{t}^{2}, p_{1} p_{2 s}, m_{t}^{2}, m_{t}^{2}, M^{2}\right) m_{t}^{4}+A_{0}\left(m_{t}^{2}\right)-A_{0}\left(M^{2}\right)\right] g_{A} g_{V} \\
& \left(p_{2}^{2}+p_{2} \cdot p_{1}\right)^{2},
\end{aligned}
$$

$$
\begin{aligned}
E_{c}= & \frac{1}{16 \pi^{2}} \frac{-1}{2\left(p_{1}^{2}+p_{1} \cdot p_{2}\right)}\left\{-2\left(B_{0}\left(p_{1} p_{2 s}, m_{t}^{2}, M^{2}\right)-B_{0}\left(m_{t}^{2}, m_{t}^{2}, M^{2}\right)\right)\left(g_{A}^{2}-g_{V}^{2}\right) m_{t}\right. \\
& +\frac{1}{m_{t}^{3}-2 m_{t}\left(p_{1}^{2}+p_{1} \cdot p_{2}\right)}\left[( g _ { A } ^ { 2 } + g _ { V } ^ { 2 } ) \left(\left(B_{0}\left(p_{1} p_{2 s}, m_{t}^{2}, M^{2}\right)-B_{0}\left(m_{t}^{2}, m_{t}^{2}, M^{2}\right)\right)\right.\right. \\
& m_{t}^{2}\left(2 m_{t}^{2}-M^{2}\right)+2\left(B_{0}\left(m_{t}^{2}, m_{t}^{2}, M^{2}\right) m_{t}^{2}-B_{0}\left(m_{t}^{2}, m_{t}^{2}, M^{2}\right) M^{2}-B_{0}\left(p_{1} p_{2 s}, m_{t}^{2}, M^{2}\right)\right. \\
& \left.\left.\left.\left.\left.m_{t}^{2}-B_{0}\left(m_{t}^{2}, m_{t}^{2}, M^{2}\right) m_{t}^{2}-A_{0}\left(m_{t}^{2}\right)+A_{0}\left(M^{2}\right)\right)\left(p_{1}^{2}+p_{1} \cdot p_{2}\right)\right)\right)\right]\right\} \\
F_{c}= & \frac{1}{16 \pi^{2}} \frac{-1}{2\left(p_{1}^{2}+p_{1} \cdot p_{2}\right)}\left\{g _ { A } g _ { V } \left[\left(B_{0}\left(p_{1} p_{2 s}, m_{t}^{2}, M^{2}\right)-B_{0}\left(m_{t}^{2}, m_{t}^{2}, M^{2}\right)\right)\left(2 m_{t}^{2}-M^{2}\right)\right.\right. \\
& \left.\left.+2\left(-1+B_{0}\left(p_{1} p_{2 s}, m_{t}^{2}, M^{2}\right)-4 C_{0}\left(0, m_{t}^{2}, p_{1} p_{2 s}, m_{t}^{2}, m_{t}^{2}, M^{2}\right) m_{t}^{2}\right)\left(p_{1}^{2}+p_{1} \cdot p_{2}\right)\right]\right\} \\
& -\delta Z_{A},
\end{aligned}
$$

$$
\begin{aligned}
G_{c}= & \frac{1}{16 \pi^{2}}\left\{C_{0}\left(0, m_{t}^{2}, p_{1} p_{2 s}, m_{t}^{2}, m_{t}^{2}, M^{2}\right)\left(g_{A}^{2}-g_{V}^{2}\right) m_{t}-\frac{1}{2\left(p_{1}^{2}+p_{1} \cdot p_{2}\right)}\left(B_{0}\left(0, m_{t}, m_{t}\right)\right.\right. \\
& \left.-B_{0}\left(p_{1} p_{2 s}, m_{t}^{2}, M^{2}\right)\right)\left(g_{A}^{2}+g_{V}^{2}\right) m_{t},
\end{aligned}
$$

$$
H_{c}=\frac{1}{16 \pi^{2}} \frac{1}{\left(p_{1}^{2}+p_{1} \cdot p_{2}\right)}\left[g_{A} g_{V}\left(B_{0}\left(p_{1} p_{2 s}, m_{t}^{2}, M^{2}\right)-B_{0}\left(m_{t}^{2}, m_{T}^{2}, m^{2}\right)\right) m_{t}\right]
$$

Where $p_{1} p_{2 s}=m_{t}-2\left(p_{1}^{2}+p_{1} \cdot p_{2}\right)$ for the off-shell $\bar{t}$ quark and $p_{1} p_{2 s}=m_{t}-2\left(p_{2}^{2}+p_{2} \cdot p_{1}\right)$ for the off-shell $t$ quark. 


\section{A4. Form factors appearing in $M_{e}$}

$$
\begin{aligned}
A_{e}= & \frac{1}{16 \pi^{2}} \frac{-1}{4\left(p_{2}^{2}+p_{2} \cdot p_{1}\right)}\left\{( g _ { A } ^ { 2 } + g _ { V } ^ { 2 } ) \left[\left(B_{0}\left(m_{t}^{2}, M^{2}, m_{t}^{2}\right)-B_{0}\left(p_{1} p_{2 s}, M^{2}, m_{t}^{2}\right)\right)\left(2 m_{t}^{2}-M^{2}\right)\right.\right. \\
& +\left(2-2 B_{0}\left(p_{1} p_{2 s}, M^{2}, m_{t}^{2}\right)+4 C_{0}\left(m_{t}^{2}+M^{2}\right)\left(m_{t}^{2}, 0, p_{1} p_{2 s}, M^{2}, m_{t}^{2}, m_{t}^{2}\right)\right) \\
& \left.\left.\frac{1}{\left(p_{2}^{2}+p_{2} \cdot p_{1}\right)}\right]\right\}-\delta Z_{V}
\end{aligned}
$$

$$
\begin{aligned}
B_{e}= & \frac{1}{16 \pi^{2}} \frac{1}{4\left[m_{t}^{2}-2\left(p_{2}^{2}+p_{2} \cdot p_{1}\right)\right]\left(p_{2}^{2}+p_{2} \cdot p_{1}\right)^{2}}\left\{\left[\left(B_{0}\left(m_{t}^{2}, M^{2}, m_{t}^{2}\right)-B_{0}\left(p_{1} p_{2 s}, M^{2}, m_{t}^{2}\right)\right)\right.\right. \\
& \left(2 m_{t}^{2}-M^{2}\right) m_{t}^{2}-2\left(B_{0}\left(m_{t}^{2}, M^{2}, m_{t}^{2}\right) m_{t}^{2}-B_{0}\left(p_{1} p_{2 s}, M^{2}, m_{t}^{2}\right) m_{t}^{2}-B_{0}^{12} M^{2}\right. \\
& -B_{0}\left(p_{1} p_{2 s}, M^{2}, m_{t}^{2}\right) M^{2}-m_{t}^{2}+B_{0}\left(m_{t}^{2}, M^{2}, m_{t}^{2}\right) m_{t}^{2}-B_{0}\left(p_{1} p_{2 s}, M^{2}, m_{t}^{2}\right) m_{t}^{2} \\
& \left.-2 C_{0}\left(m_{t}^{2}, 0, p_{1} p_{2 s}, M^{2}, m_{t}^{2}, m_{t}^{2}\right) m_{t}^{4}-A_{0}\left(m_{t}^{2}\right)-A_{0}\left(M^{2}\right)\right)\left(p_{2}^{2}+p_{2} \cdot p_{1}\right) \\
& \left.\left.-4\left(1+2 C_{0}\left(m_{t}^{2}, 0, p_{1} p_{2 s}, M^{2}, m_{t}^{2}, m_{t}^{2}\right) m_{t}^{2}\right)\left(p_{2}^{2}+p_{2} \cdot p_{1}\right)^{2}\right]\left(g_{A}^{2}+g_{V}^{2}\right)\right\}
\end{aligned}
$$

$$
\begin{aligned}
C_{e}= & \frac{1}{16 \pi^{2}} \frac{-1}{m_{t}\left[m_{t}^{2}-2\left(p_{2}^{2}+p_{2} \cdot p_{1}\right)\right]\left(p_{2}^{2}+p_{2} \cdot p_{1}\right)}\left\{g _ { A } g _ { V } \left[\left(B_{0}\left(m_{t}^{2}, M^{2}, m_{t}^{2}\right)\right.\right.\right. \\
& \left.-B_{0}\left(p_{1} p_{2 s}, M^{2}, m_{t}^{2}\right)\right)\left(2 m_{t}^{2}-M^{2}\right) m_{t}^{2}-2\left(B_{0}\left(m_{t}^{2}, M^{2}, m_{t}^{2}\right) m_{t}^{2}-B_{0}\left(m_{t}^{2}, M^{2}, m_{t}^{2}\right) M^{2}\right. \\
& \left.+A_{0}\left(M^{2}\right)+B_{0}\left(m_{t}^{2}, M^{2}, m_{t}^{2}\right) m_{t}^{2}-B_{0}\left(p_{1} p_{2 s}, M^{2}, m_{t}^{2}\right) m_{t}^{2}-A_{0}\left(m_{t}^{2}\right)\right) \\
& \left.\left.\left(p_{2}^{2}+p_{2} \cdot p_{1}\right)\right]\right\}
\end{aligned}
$$

$$
\begin{aligned}
D_{e}= & \frac{1}{16 \pi^{2}} \frac{-1}{2\left[m_{t}^{2}-2\left(p_{2}^{2}+p_{2} \cdot p_{1}\right)\right]\left(p_{2}^{2}+p_{2} \cdot p_{1}\right)^{2}}\left\{\left[\left(B_{0}\left(m_{t}^{2}, M^{2}, m_{t}^{2}\right)-B_{0}\left(p_{1} p_{2 s}, M^{2}, m_{t}^{2}\right)\right)\right.\right. \\
& \left(2 m_{t}^{2}-M^{2}\right) m_{t}^{2}-2\left(B_{0}\left(m_{t}^{2}, M^{2}, m_{t}^{2}\right) m_{t}^{2}-B_{0}\left(p_{1} p_{2 s}, M^{2}, m_{t}^{2}\right) m_{t}^{2}\right. \\
& +B_{0}\left(m_{t}^{2}, M^{2}, m_{t}^{2}\right) M^{2}+B_{0}\left(p_{1} p_{2 s}, M^{2}, m_{t}^{2}\right) M^{2}-m_{t}^{2}+B_{0}\left(m_{t}^{2}, M^{2}, m_{t}^{2}\right) m_{t}^{2} \\
& \left.\left.-B_{0}\left(p_{1} p_{2 s}, M^{2}, m_{t}^{2}\right) m_{t}^{2}-2 C_{0}\left(m_{t}^{2}, 0, p_{1} p_{2 s}, M^{2}, m_{t}^{2}, m_{t}^{2}\right) m_{t}^{4}+A_{0}\left(m_{t}^{2}\right)-A_{0} M^{2}\right)\right) \\
& \left.\left.\left(p_{2}^{2}+p_{2} \cdot p_{1}\right)-4\left(1+2 C_{0}\left(m_{t}^{2}, 0, p_{1} p_{2 s}, M^{2}, m_{t}^{2}, m_{t}^{2}\right) m_{t}^{2}\right)\left(p_{2}^{2}+p_{2} \cdot p_{1}\right)^{2}\right] g_{A} g_{V}\right\},
\end{aligned}
$$




$$
\begin{aligned}
E_{e}= & \frac{1}{8 \pi^{2}} C_{0}\left(m_{t}^{2}, 0, p_{1} p_{2 s}, M^{2}, m_{t}^{2}, m_{t}^{2}\right) 2 g_{V}^{2} m_{t}+\left[( - g _ { A } ^ { 2 } m _ { t } - 3 g _ { V } ^ { 2 } m _ { t } ) \left(B_{0}\left(m_{t}^{2}, M^{2}, m_{t}^{2}\right)-B_{0}\right.\right. \\
& \left.\left.\left(p_{1} p_{2 s}, M^{2}, m_{t}^{2}\right)-2 C_{0}\left(m_{t}^{2}, 0, p_{1} p_{2 s}, M^{2}, m_{t}^{2}, m_{t}^{2}\right)\left(p_{2}^{2}+p_{2} \cdot p_{1}\right)\right)\right] \\
& +\frac{1}{16 \pi^{2}} \frac{1}{\left(p_{2}^{2}+p_{2} \cdot p_{1}\right)}\left\{( g _ { A } ^ { 2 } + g _ { V } ^ { 2 } ) m _ { t } \left[B_{0}\left(m_{t}^{2}, M^{2}, m_{t}^{2}\right)-B_{0}\left(p_{1} p_{2 s}, M^{2}, m_{t}^{2}\right)\right.\right. \\
& +\frac{B_{0}\left(m_{t}^{2}, M^{2}, m_{t}^{2}\right)\left(-M^{2}\right)+A_{0}\left(m_{t}^{2}\right)+A_{0}\left(M^{2}\right)}{2 m_{t}^{2}} \\
& +2 C_{0}\left(m_{t}^{2}, 0, p_{1} p_{2 s}, M^{2}, m_{t}^{2}, m_{t}^{2}\right)\left(p_{2}^{2}+p_{2} \cdot p_{1}\right) \\
& +\frac{\left.\left.-A_{0}\left(m_{t}^{2}\right)+A_{0}\left(M^{2}\right)+B_{0}\left(p_{1} p_{2 s}, M^{2}, m_{t}^{2}\right)\left(-M^{2}+2\left(p_{2}^{2}+p_{2} \cdot p_{1}\right)\right)\right]\right\}}{2\left[m_{t}^{2}-2\left(p_{2}^{2}+p_{2} \cdot p_{1}\right)\right]}
\end{aligned}
$$

$$
\begin{aligned}
F_{e}= & \frac{1}{32 \pi^{2}\left(p_{2}^{2}+p_{2} \cdot p_{1}\right)}\left\{g _ { A } g _ { V } \left[\left(B_{0}\left(m_{t}^{2}, M^{2}, m_{t}^{2}\right)-B_{0}\left(p_{1} p_{2 s}, M^{2}, m_{t}^{2}\right)\right)\left(2 m_{t}^{2}-M^{2}\right)+4 C_{0}\right.\right. \\
& \left.\left(m_{t}^{2}, 0, p_{1} p_{2 s}, M^{2}, m_{t}^{2}, m_{t}^{2}\right)\left(m_{t}^{2}+M^{2}\right)\left(p_{2}^{2}+p_{2} \cdot p_{1}\right)+\left(2-2 B_{0}\left(p_{1} p_{2 s}, M^{2}, m_{t}^{2}\right)\right]\right\} \\
& -\delta Z_{A},
\end{aligned}
$$

$$
\begin{aligned}
G_{e}= & \frac{m_{t}}{32 \pi^{2}\left(p_{2}^{2}+p_{2} \cdot p_{1}\right)}\left(B_{0}\left(m_{t}^{2}, M^{2}, m_{t}^{2}\right)-B_{0}\left(p_{1} p_{2 s}, M^{2}, m_{t}^{2}\right)\right)\left(g_{A}^{2}+g_{V}^{2}\right)+\left(p_{2}^{2}+p_{2} \cdot p_{1}\right) \\
& 2 C_{0}\left(m_{t}^{2}, 0, p_{1} p_{2 s}, M^{2}, m_{t}^{2}, m_{t}^{2}\right) m_{t}\left(g_{A}^{2}-g_{V}^{2}\right)
\end{aligned}
$$

$$
\begin{aligned}
H_{e}= & \frac{1}{16 \pi^{2}} \frac{1}{\left(p_{2}^{2}+p_{2} \cdot p_{1}\right)^{2}} g_{A} g_{V} m_{t}\left[2\left(B_{0}\left(m_{t}^{2}, M^{2}, m_{t}^{2}\right)-B_{0}\left(p_{1} p_{2 s}, M^{2}, m_{t}^{2}\right)\right) m_{t}^{2}\right. \\
& +\left(B_{0}\left(m_{t}^{2}, M^{2}, m_{t}^{2}\right)-B_{0}\left(0, m_{t}^{2}, m_{t}^{2}\right)-2 C_{0}\left(m_{t}^{2}, 0, p_{1} p_{2 s}, M^{2}, m_{t}^{2}, m_{t}^{2}\right) M^{2}\right) M^{2}\left(p_{2} \cdot p_{1}\right. \\
& \left.\left.+p_{2}^{2}\right)+2 C_{0}\left(m_{t}^{2}, 0, p_{1} p_{2 s}, M^{2}, m_{t}^{2}, m_{t}^{2}\right)+B_{0}\left(p_{1} p_{2 s}, M^{2}, m_{t}^{2}\right)\right] .
\end{aligned}
$$

\section{A5. Form factors appearing in $M_{i}$}

$$
\begin{aligned}
A_{i}= & 4 g_{V} m_{t}\left\{\left[m_{t}^{2}-\left(p_{1}+p_{2}\right)^{2}+\left(p_{1}^{2}+p_{1} \cdot p_{2}\right)\right] C_{0}\left(p_{1}^{2},\left(p_{2}-p_{1}\right)^{2}, 0, m_{t}^{2}, m_{t}^{2}\right)\right. \\
& {\left[\left(p_{1}-3 p_{2}\right)\left(p_{2}-p_{1}\right)\right] C_{12}\left(p_{1}^{2},\left(p_{2}-p_{1}\right)^{2}, 0, m_{t}^{2}, m_{t}^{2}\right)-\left(3 p_{1}^{2}+p_{1} \cdot p_{2}\right) } \\
& C_{11}\left(p_{1}^{2},\left(p_{2}-p_{1}\right)^{2}, 0, m_{t}^{2}, m_{t}^{2}\right)+2\left(p_{1} \cdot p_{2}-p_{1}^{2}\right) C_{11}\left(p_{1}^{2},\left(p_{2}-p_{1}\right)^{2}, 0, m_{t}^{2}, m_{t}^{2}\right) \\
& C_{12}\left(p_{1}^{2},\left(p_{2}-p_{1}\right)^{2}, 0, m_{t}^{2}, m_{t}^{2}\right)-p_{1}^{2} C_{11}^{2}\left(p_{1}^{2},\left(p_{2}-p_{1}\right)^{2}, 0, m_{t}^{2}, m_{t}^{2}\right),
\end{aligned}
$$




$$
\begin{aligned}
B_{i} & =16 m_{t} g_{V} C_{22}\left(p_{1}^{2},\left(p_{2}-p_{1}\right)^{2}, 0, m_{t}^{2}, m_{t}^{2}\right) \\
C_{i} & =8 m_{t} g_{V}\left[2 C_{23}\left(p_{1}^{2},\left(p_{2}-p_{1}\right)^{2}, 0, m_{t}^{2}, m_{t}^{2}\right)-C_{12}\left(p_{1}^{2},\left(p_{2}-p_{1}\right)^{2}, 0, m_{t}^{2}, m_{t}^{2}\right)\right] \\
D_{i} & =8 m_{t} g_{V} C_{12}\left(p_{1}^{2},\left(p_{2}-p_{1}\right)^{2}, 0, m_{t}^{2}, m_{t}^{2}\right) \\
E_{i} & =16 m_{t} g_{V} C_{12}\left(p_{1}^{2},\left(p_{2}-p_{1}\right)^{2}, 0, m_{t}^{2}, m_{t}^{2}\right) \\
F_{i} & =-16 m_{t} g_{V} C_{11}\left(p_{1}^{2},\left(p_{2}-p_{1}\right)^{2}, 0, m_{t}^{2}, m_{t}^{2}\right) \\
G_{i} & =8 m_{t} g_{V} C_{0}\left(p_{1}^{2},\left(p_{2}-p_{1}\right)^{2}, 0, m_{t}^{2}, m_{t}^{2}\right) \\
H_{i} & =-4 i m_{t} g_{A} C_{0}\left(p_{1}^{2},\left(p_{2}-p_{1}\right)^{2}, 0, m_{t}^{2}, m_{t}^{2}\right) \\
I_{i} & =-4 m_{t} g_{V} C_{0}\left(p_{1}^{2},\left(p_{2}-p_{1}\right)^{2}, 0, m_{t}^{2}, m_{t}^{2}\right) \\
J_{i} & =4 i m_{t} g_{A} C_{0}\left(p_{1}^{2},\left(p_{2}-p_{1}\right)^{2}, 0, m_{t}^{2}, m_{t}^{2}\right) .
\end{aligned}
$$

A6. The counterterms $\delta Z_{V}, \delta Z_{A}$, and $\delta m_{t}$

$$
\begin{aligned}
& \delta Z_{A}=\frac{1}{16 \pi^{2}} \frac{g_{A} g_{V}}{m_{t}^{2}}\left[A_{0}\left(M^{2}\right)-A_{0}\left(m_{t}^{2}\right)+\left(2 m_{t}^{2}-M^{2}\right) B_{0}\left(m_{t}^{2}, M^{2}, m_{t}^{2}\right)\right], \\
& \delta Z_{V}=\frac{1}{16 \pi^{2}} \frac{g_{A}^{2}+g_{V}^{2}}{2 m_{t}^{2}}\left[A_{0}\left(M^{2}\right)-A_{0}\left(m_{t}^{2}\right)+\left(2 m_{t}^{2}-M^{2}\right)\right] \\
& B_{0}\left(m_{t}^{2}\right)\left(m_{t}^{2}, M^{2}, m_{t}^{2}\right)+\frac{1}{16 \pi^{2}}\left[\left(g_{A}^{2}+g_{V}^{2}\right)\left(M^{2}-2 m_{t}^{2}\right)\right. \\
&\left.-\left(g_{A}^{2}-g_{V}^{2}\right) 2 m_{t}^{2}\right] B_{0}^{\prime}\left(m_{t}^{2}\right), \\
& \delta m_{t}=-\frac{1}{16 \pi^{2}}\left[\left(4 m_{t}^{2}-M^{2}\right)\left(g_{A}^{2}+g_{V}^{2}\right)\right] B_{0}^{\prime}\left(m_{t}^{2}\right)+\frac{1}{16 \pi^{2} m_{t}^{2}} \\
&\left\{\left(g_{A}^{2}+g_{V}^{2}\right)\left[A_{0}(M)^{2}-A_{0}\left(m_{t}^{2}\right)\right]+\left[\left(g_{A}^{2}+g_{V}^{2}\right)\right.\right. \\
&\left.\left.\left(m_{t}^{2}-M^{2}\right)+\left(g_{A}^{2}-g_{V}^{2}\right) m_{t}^{2}\right] B_{0}\left(m_{t}^{2}, M^{2}, m_{t}^{2}\right)\right\} .
\end{aligned}
$$




\section{References}

[1] For a recent review, see: N. E. Adam et al, arXiv: 0803.1154 [hep-ph].

[2] C. T. Hill and E. H. Simmons, Phys. Rept. 381 (2003)235; [Erratum-ibid, (2004)390,553].

[3] For recent review, see: W. Bernreuther, J. Phys. G35 (2008)083001; J. R. Incandela, A. Quadt, W. Wagner, D. Wicke, Prog. Part. Nucl. Phys. 63 (2009)239.

[4] T. Stelzer and S. Willenbrock, Phys. Lett. B374 (1996)169; A. Brandenburg, Phys. Lett. B388 (1996)626; D. Chang, S. C. Lee and A. Sumarokov, Phys. Rev. Lett. 77 (1996)1218.

[5] V. D. Barger, J. Ohnemus and R. J. N. Phillips, Int. J. Mod. Phys. A4 (1989)617; T. Arens and L. M. Sehgal, Phys. Lett. B302 (1993)501; G. Mahlon and S. J. Parke, Phys. Rev. D53 (1996)4886; Phys. Lett. B411 (1997)173.

[6] W. Bernreuther, A. Brandenburg, Z. G. Si and P. Uwer, Phys. Rev. Lett. 87 (2001)242002; Nucl. Phys. B690 (2004)81; Peter Uwer, Phys. Lett. B609 (2005)271.

[7] K. Y. Lee, H. S. Song, J. H. Song and C. Yu, Phys. Rev. D60 (1999)093002; K. Y. Lee, S. C. Park, H. S. Song and C. Yu, Phys. Rev. D63 (2001)094010; C. X. Yue, L. N. Wang, J. Phys. G34 (2007)139; B. Sahin, arXiv: 0802.1937 [hep-ph].

[8] K. Y. Lee, S. C. Park, H. S. Song, J. H. Song and C. Yu, Phys. Rev. D61 (2000)074005; I. Sahin, Eur. Phys. J. C60 (2009)431.

[9] King-Man Cheung, Phys. Rev. D55 (1997)4430; B. Holdom, T. Torma, Phys. Rev. D60 (1999)114010; M. Arai, N. Okada, K. Smolek and V. Simak, Phys. Rev. D70 (2004)115015; Phys. Rev. D75 (2007)095008; Acta. Phys. Polon. B40 (2009)93; M. Arai, N. Okada, K. Smolek, Phys. Rev. D79 (2009)074019; J. Y. Liu, Z. G. Si and C. X. Yue, Phys. Rev. D81 (2010)015011. 
[10] C. T. Hill, Phys. Lett. B345 (1995)483; K. D. Lane and E. Eichten, Phys. Lett. B352 (1995)382; K. D. Lane, Phys. Lett. B433 (1998)96; G. Cvetic, Rev. Mod. Phys. 71 (1999)513.

[11] G. Buchalla, G. Burdman, C. T. Hill, D. Kominis, Phys. Rev. D53 (1996)5185.

[12] W. Loniaz, T.Takuch, Phys. Rev. D62 (1999)055005.

[13] H. J. He, C. P. Yuan, Phys. Rev. Lett. 83 (1999)28; G. Burdman, Phys. Rev. Lett. 83 (1999)2888; H. J. He, S. Kanemura, C. P. Yuan, Phys. Rev. Lett. 89 (2002)101803.

[14] A. K. Leibovich and D. L. Rainwater, Phys. Rev. D65 (2002)055012.

[15] G. Passarino and M. J. G. Veltman, Nucl. Phys. B160 (1979)151.

[16] J. Pumplin, et al. (CTEQ Collaboration), JHEP 0602 (2006)032.

[17] A. Czarnecki, M. Jezabek and J. H. Kuhn, Nucl. Phys. B351 (1991)70; A. Brandenburg, Z. G. Si and P. Uwer, Phys. Lett. B539 (2002)235.

[18] W. M. Yao et al. [Particle Data Group], J. Phys. G33 (2006)1 and partial updat for the 2008 edition.

[19] R. S. Chivukula, B. Dobrescu, H. Georgi, C. T. Hill, Phys. Rev. D59 (1999)075003.

[20] B. Balaji, Phys. Lett. B393 (1997)89.

[21] G. Burdman and D. Kominis, Phys. Lett. B403 (1997)101; C. X.Yue, Y. P. Kuang, X. L. Wang and W. B. Li, Phys. Rev. D62 (2000)055005.

[22] F. Hubaut, E. Monnier, P. Pralavorio, V. Simak, K. Smolek, Eur. Phys. J. C44(2005)13. 